\title{
3D MODELING OF COMPONENTS OF A GARDEN BY USING POINT CLOUD DATA
}

\author{
R. Kumazakia*, Y. Kunii a \\ aDepartment of Landscape Architecture Science, Tokyo University of Agriculture, \\ 1-1-1 Sakuragaoka, Setagaya, Tokyo, 156-8502, Japan (45715004, y3kunii)@ nodai.ac.jp
}

Commission V, WG V/2

KEY WORDS: Laser measurement, point cloud data, Japanese garden, landscape material, 3D model library

\begin{abstract}
:
Laser measurement is currently applied to several tasks such as plumbing management, road investigation through mobile mapping systems, and elevation model utilization through airborne LiDAR. Effective laser measurement methods have been well-documented in civil engineering, but few attempts have been made to establish equally effective methods in landscape engineering. By using point cloud data acquired through laser measurement, the aesthetic landscaping of Japanese gardens can be enhanced. This study focuses on simple landscape simulations for pruning and rearranging trees as well as rearranging rocks, lanterns, and other garden features by using point cloud data. However, such simulations lack concreteness. Therefore, this study considers the construction of a library of garden features extracted from point cloud data. The library would serve as a resource for creating new gardens and simulating gardens prior to conducting repairs. Extracted garden features are imported as 3ds Max objects, and realistic 3D models are generated by using a material editor system. As further work toward the publication of a 3D model library, file formats for tree crowns and trunks should be adjusted. Moreover, reducing the size of created models is necessary. Models created using point cloud data are informative because simply shaped garden features such as trees are often seen in the 3D industry.
\end{abstract}

\section{INTRODUCTION}

3D laser scanners are currently used in plumbing maintenance and operations, road condition surveying with mobile mapping systems (MMS), and topographic surveying with airborne LiDAR data. The use of 3D laser scanners is especially welldocumented in civil engineering, in which effective scanning methods have been established.

Within the various applications of 3D laser scanners, a close analysis of trees gathered from 3D point cloud data has been done to compare the structures of trees using relative differences that are characterized by geometric forms to remove the trees that are constructed from point cloud data in MMS (Xin, 2014). The use of airborne LiDAR for mapping forests has been widely documented. With LiDAR, it is possible to discriminate tree species on the basis of crown shapes and to understand the phytogeography of forests. However, very few attempts have been made at creating a 3D model to obtain detailed structures of trees from acquired data.

From the perspective of landscape architecture, the most important aspect of a Japanese garden is planting design because trees effectively emphasize symbolism and abstractness (Kyozo, 1984). A study involving the characteristics of landscape planting made several important statements on trees. According to the study, the three components of tree size, tree number, and vegetation coverage may account for as much as $76 \%$ of the characteristics of landscape planting (Kyozo, 1984). Furthermore, the regularity surrounding the planting of trees in a Japanese garden is reflected by the location of the garden and the tree species used while the scale of a garden is related to the scale of the trees used. The individual characteristics of tree scale are particular to each Japanese garden (Kyozo, 1986). Therefore, trees are clearly an important scenic feature in Japanese gardens.

For this reason, in order to demonstrate the process for extracting features such as trees and stones from 3D point cloud data, visualizing and understanding those features in the context of a Japanese garden are important. Consequently, it is reasonable to expect that a library built on the basis of $3 \mathrm{D}$ models would be continuously updated with landscape features.

\section{RESEARCH CONTENTS}

This study attempts to use 3D point cloud data gathered from four Japanese gardens (Table 1). 3D point cloud data can be used for a garden's master plan, and ortho-planes and contour lines can be extracted from the data. The extracted contour lines make drawing a ground plan possible. Consequently, the drawing and extraction of the contour lines of a Japanese garden could be achieved more efficiently (Rihito, 2015).

However, using some landscape simulations may result in unsatisfactory recommendations such as the removal or cutting of trees to create new sightseeing spots, as seen in the tree cutting simulation at Odawara Castle (Figure 1).

Accordingly, this study suggests an effective method for extracting landscape features from 3D point cloud data. Extracted 3D point cloud data are considered informative for the field of tree design because the data can precisely express the shape of landscape features. In addition, the leaves, branches, and trunks of trees have complicated surface shapes

\footnotetext{
* Corresponding author
} 


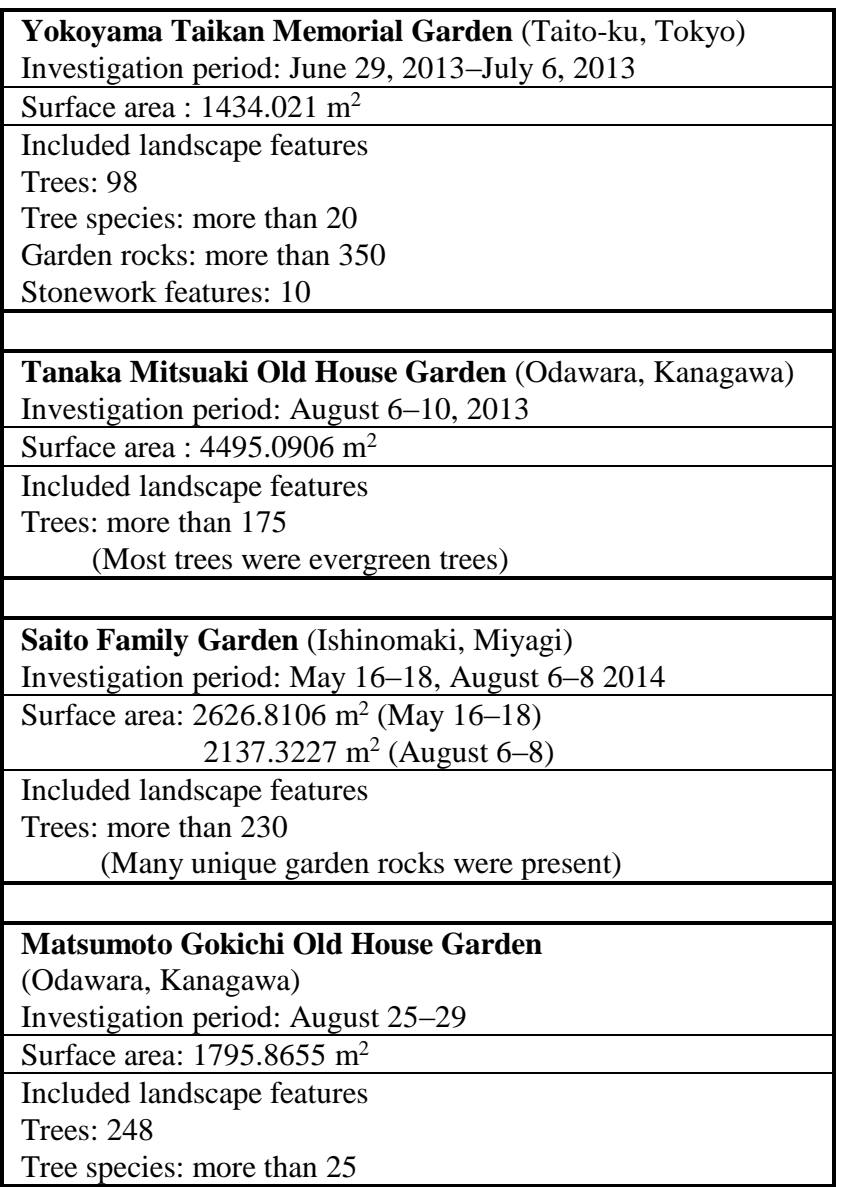

Table 1. Measurement area and number of garden features

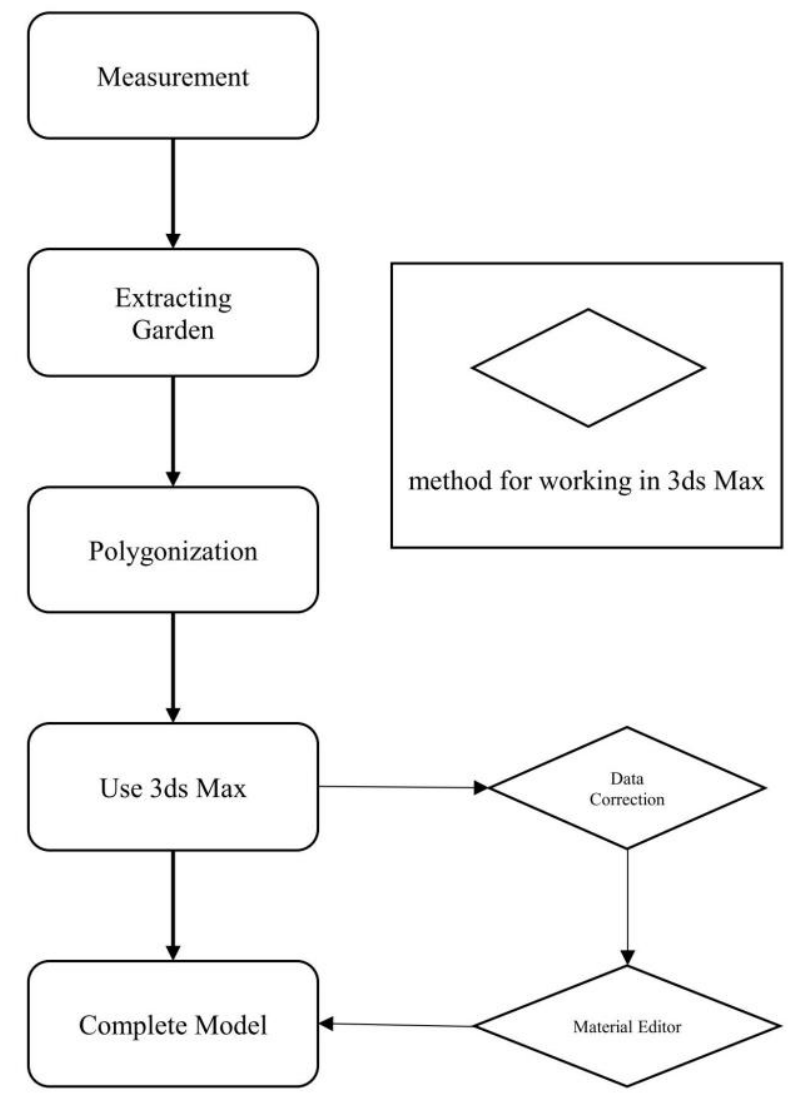

Figure 2. Flowchart of production process for tree modeling
(Atsushi, 2014). Consequently, creating 3D models of landscape features by extracting 3D point cloud data from Japanese gardens is considered informative and demonstrates that the process can distinguish between tree species and make 3D models from extracting 3D point cloud data.

\section{PRODUCTION PROCESS FOR TREE MODELING}

A flowchart of the production method is shown in Figure 2.

\subsection{Extracting Trees}

The first step in extracting a tree is understanding the shape of the trunk (Figure 3). Most coniferous trees such as red pines have nearly circular trunks, and the forms of the trunks and shapes of the trees are affected by the tree branches that grow radially from the centers of the trunks (Hayashi, 1997). Consequently, extracting the shape of the tree trunk seems to be the most important part of $3 \mathrm{D}$ modeling for distinguishing tree species.

\subsection{Mesh Generation of Tree Trunks}

This 3D model is a generated polygon mesh of part of a trunk based on a red pine extracted from 3D point cloud data by using "mesh lab" (Figure 4). Generating a polygon mesh of branches and leaves is difficult because they do not have extended outer surfaces. A polygon model of the trunk data is then exported to OBJ format in order to import it in $3 \mathrm{ds}$ Max

\subsection{Using 3ds Max}

3.3.1 Revising 3D Trunk Model: In order to depict deformed branches in trunk polygon models that are exported to OBJ format, correcting the deformities is necessary. To do this, it is essential to create branches that have not been generated in the mesh generation stage. Using "Autodesk Recap 360" enables the importing of 3D point cloud data for 3ds Max by converting the data to RCS format. Accordingly, a complete 3D trunk model can be created by superimposing trunk 3D point cloud data on the 3D trunk model (Figure 5).

3.3.2 Using Material Editor for 3ds Max: Using the material editor for $3 \mathrm{ds}$ Max enables detailed texture creation. In addition, physical texture can be expressed by using a displacement map and a bump map in the material editor other than a set texture for the 3D trunk model of a red pine (Figure $6)$.

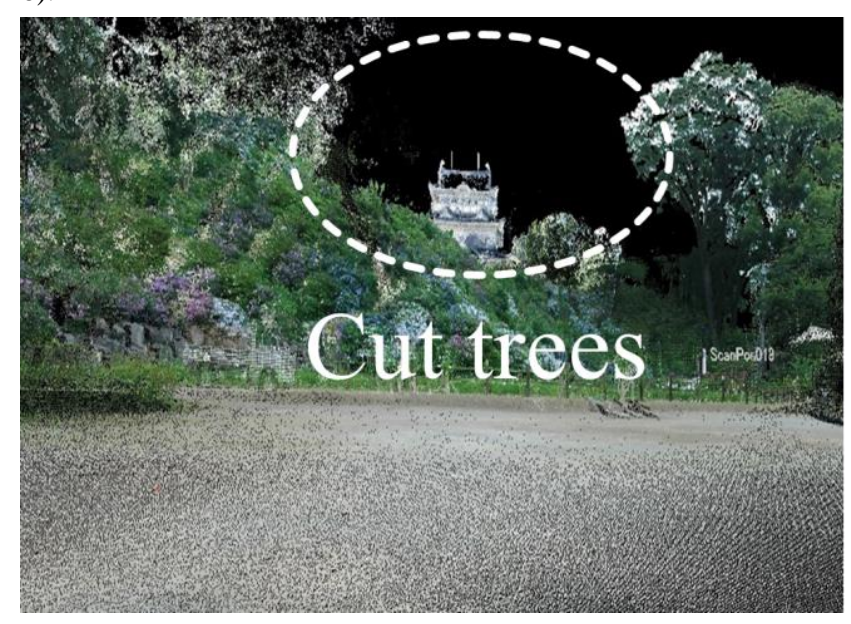

Figure 1. Tree cutting simulation at Odawara Castle 


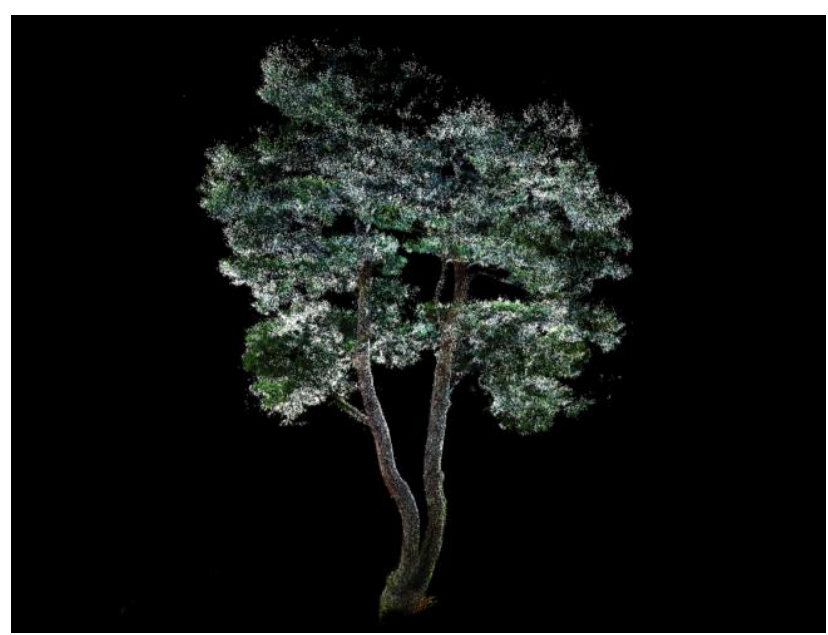

Figure 3. Extracted red pine tree

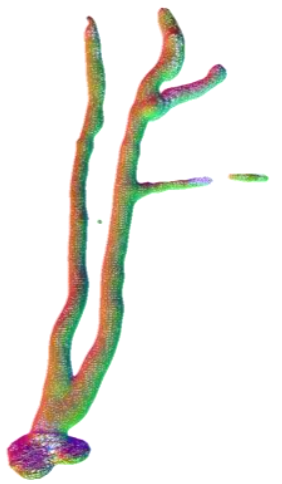

Figure 4. Generated polygon mesh of trunk

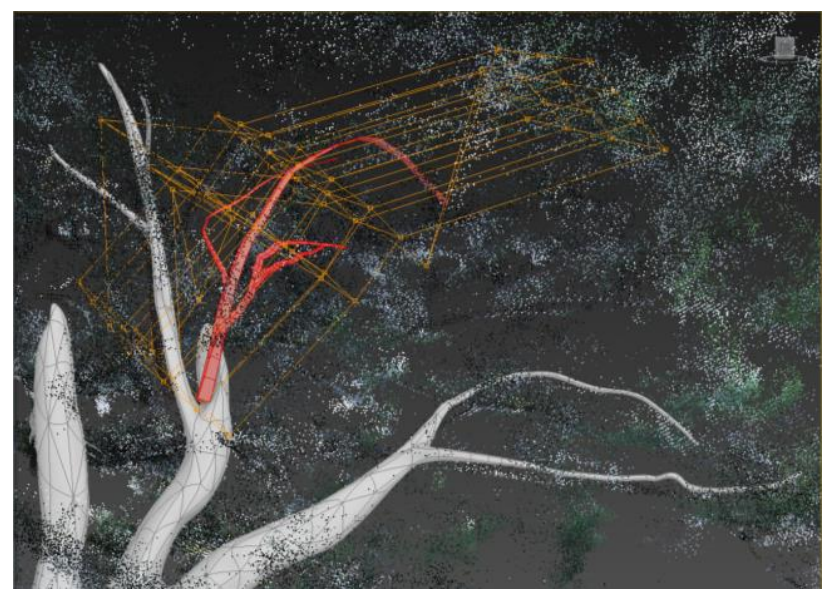

Figure 5. Revision of 3D trunk mode

3.3.3 Successful Red Pine Model: A 3D model of a red pine was completed by adding leaves expressed in 3D point cloud data (Figure 7). While the intricate details of the peeled, "tortoise-shell" textured red pine bark could not be expressed, the characteristic red appearance of the bark along with its deep cracks could be rendered. Tree conditions provide a valuable record of past storm damage, changes to the landscape, and an estimation of insolation (Akiyo, 1984) (Kikumatsu, 1981). These conditions were reflected in the red pine model. Thus, it can be inferred that using $3 \mathrm{D}$ point cloud data enables the creation of realistic tree forms.

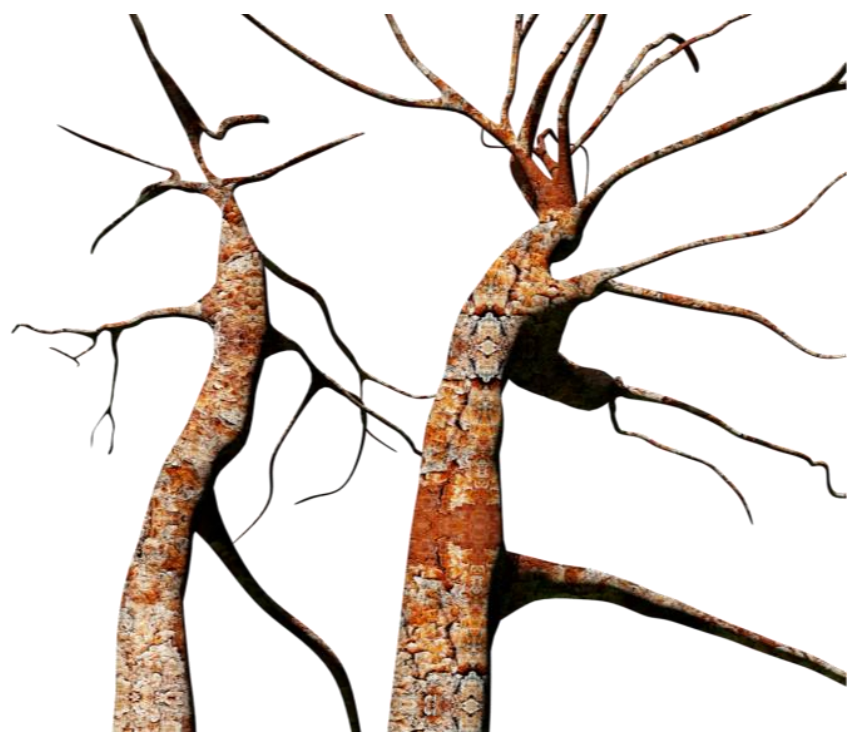

Figure 6. Red pine trunk model

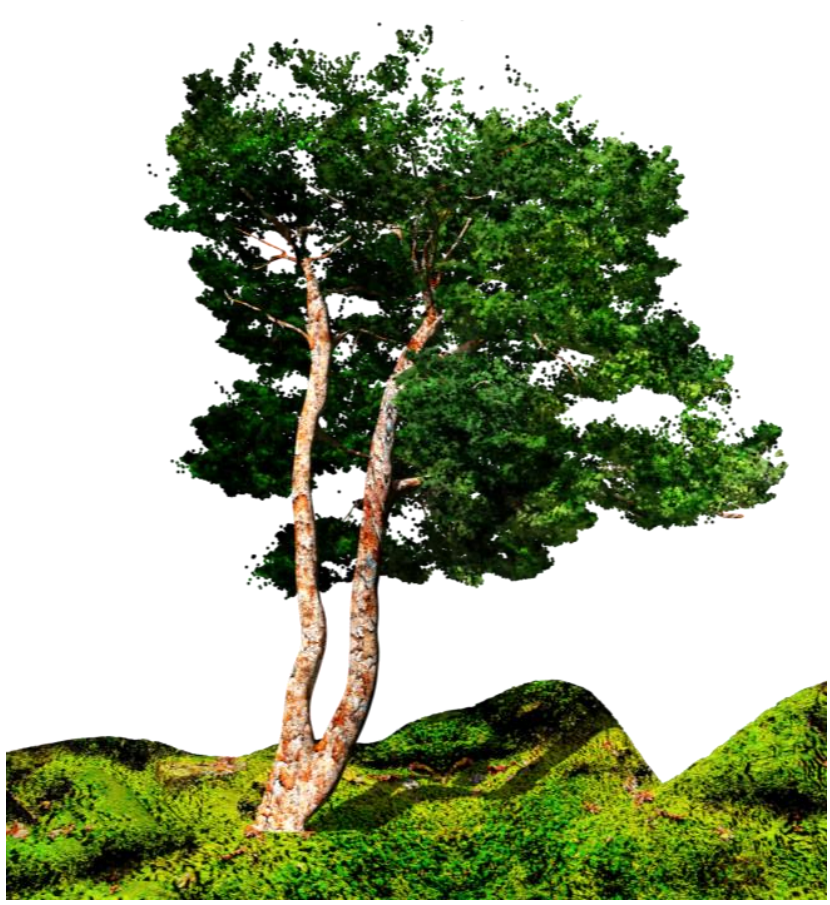

Figure 7. Successful Red Pine Model

3.3.4 Issues with Red Pine Model: While the trunk of the red pine could be accurately rendered in the model, the leaves were less detailed because it is impossible to accurately express acerose leaf characteristics in 3D point cloud data. In order to solve this problem, advancements in the polygon 3D modeling of acerose leaves need to be model.

\section{MODELING THE BLACK PINE OF SHIBAMATA TAISHAKUTEN}

A 3D model of the symbolic black pine of Shibamata Taishakuten in Katsushika-ku, Tokyo, was made. The trunk's close proximity to the ground made detailed measurements difficult. However, it was possible to render the trunk as well as that of the red pine. 3D point cloud data could be extracted in the same fashion as with the red pine, and a 3D model of the black pine was created (Figures 8,9). 


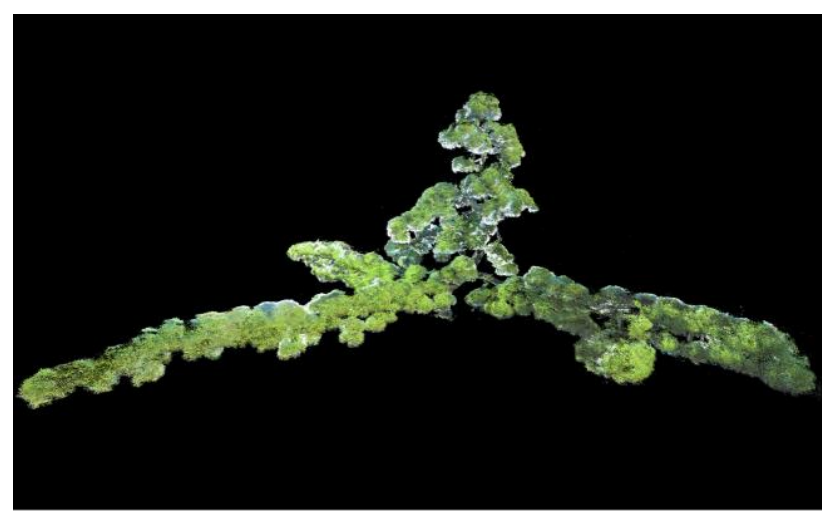

Figure 8. Black pine data from 3D point cloud

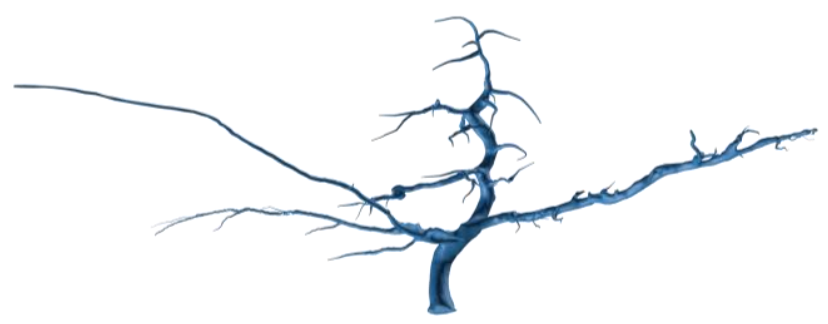

Figure 9. Polygon model of black pine trunk

\subsection{Creation of Leaves}

In order to realistically render leaves with polygons, each acerose leaf was expressed using one polygon. Using more than one polygon would result in display speed delays caused by the large amount of data involved in creating leaves with curved surfaces and multiple vertexes.

4.1.1 Creation of Acerose Leaves: As a first step in expressing realistic acerose leaves, enormous polygons and vertexes were shaped into leaves. The entire leaf model for the tree consisted of 2814 vertexes and 3284 polygons (Figure 10).

4.1.2 Expression by Two Polygons: Rendering is performed for each side of an acerose leaf, and the resulting 3D model is then exported to a Targa format supporting an alpha channel. Next, the Targa formatted 3D model of the acerose leaf is exported to $3 \mathrm{ds}$ Max, where map textures are layered onto polygons by using a material editor equipped with a bitmap tool In addition, asymmetrical expression is possible by using a double-sided map when rendering each side of a leaf (Figure $11)$.

\subsection{Attachment of Leaves and Successful Black Pine Model}

The acerose leaf model is attached to the branch model with two polygons by using the object paint tool in $3 \mathrm{ds}$ Max. The width of the acerose leaf model needs to be adjusted before being attached to the branch model. Moreover, a paint tool object list is added to both the leaf and branch models, which are attached in random order. The completed branch model is then attached to the trunk model in the same way. Finally, the completed black pine leaf model is compared to the arrangement of leaves on an actual stem on the basis of 47 pictures taken in Shibamata Taishakuten (Figure 12)

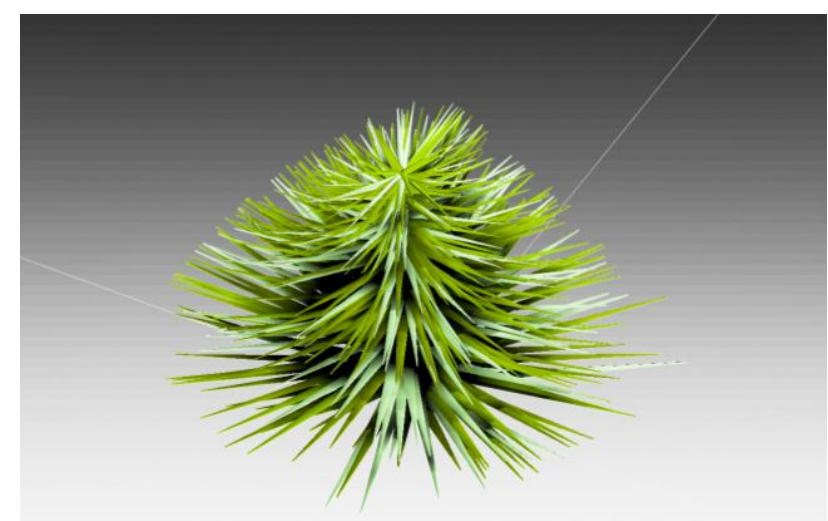

Figure 10. 3D model of acerose leaf

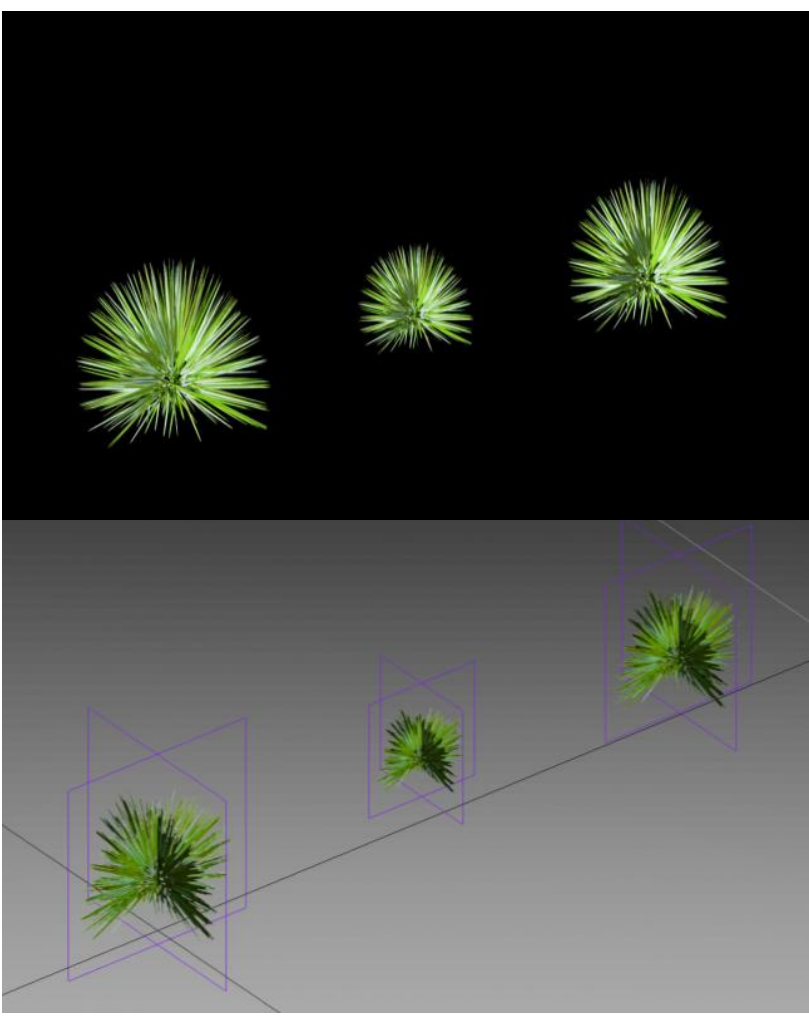

Figure 11. Expression by two polygons

\section{APPLICATION TO DIFFERENT TREE MODELS}

In this study, tree models were created on the basis of 3D point cloud data, and the appearance of tree trunks could be faithfully rendered. This model creation method has distinctive features. The method takes into account the influence of wind, insolation, and the surrounding landscape on tree forms. Tree forms are also affected by tree growth and environmental conditions, and different tree species take on different forms. Thus far, tree forms have shown to be the most important feature in $3 \mathrm{D}$ modeling for Japanese gardens. However, the method for creating 3D models of trees has clearly proven to consume lots of time and labor. For this reason, deriving new tree models from existing tree models is necessary. The maple tree model in this study was derived from the red pine model. The bushy crown of the red pine model is very similar to that of the maple, enabling the creation of the maple by making the necessary changes in texture and form (Figure 13). 


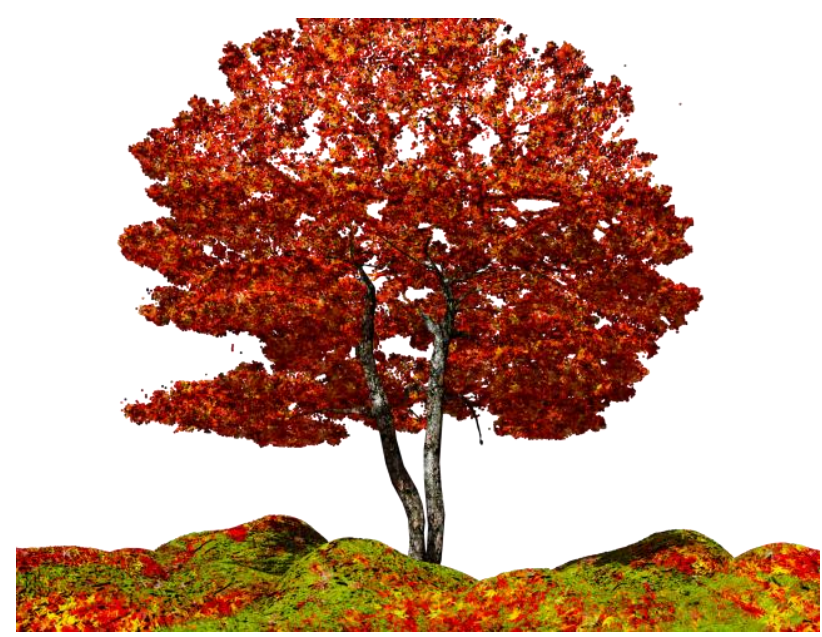

Figure 13. Application to deciduous tree model

\section{RESULTS AND DISCUSSION}

This study focuses on the creation of $3 \mathrm{D}$ models of garden features by using 3D point cloud data. Emphasis is placed on trees because they are the most important features of Japanese gardens. The creation of 3D models takes a considerable amount of time due to the complexity of tree shapes and the demand for realistic depictions (Takashi, 2014).

Currently, 3D point cloud data has been used for Japanese gardens to create grand plans, investigate landscape planting, and to remove, rearrange, or prune trees, as shown in the tree cutting simulation. The method of 3D model creation using features extracted from 3D point cloud data has proven to be beneficial.

Consequently, the construction of a library for 3D models of Japanese garden features extracted from 3D point cloud data is expected. However, the software environment for creating 3D models is currently limited to $3 \mathrm{~d}$ s Max. Moreover, reducing the size of created 3D models will be necessary in the future.

\section{REFERENCES}

Xin, Z., Shunsuke, A., Kenichi, K., 2014. An Extracting Method of Building's Walls from MMS Point Cloud against Influences of Trees. GIS Association of Japan, E-6-1

Kyozo, C., Toshikatsu, H., 1984. A Study on Characteristics of Landscape Planting of Woody Plants in Japanese Gardens.

Kyozo, C., Toshikatsu, H., 1986. Compositional Characteristics of Woody Plants and Rocks Used in Japanese Gardens and an Application of Them to the Computer Aided Garden Design System.

Rihito, K., Yoichi, K., 2015. Drawing and Landscape Simulation for Garden by Using Terrestrial Laser Scanner. Journal of Agriculture Science, Tokyo University of Agriculture, Vol. 60, No. 2, 93-102

Atsushi, M., Kazunori, M., 2014. Modeling Trees Using Surface Growth Simulation. The Journal of the Society for Art and Science, Vol. 13, No. 1, pp. 45-58

Hayashi, S., Takahashi, Y., 1997. On the modeling of a tree's figure - Case of Cryptomeria japonica and Chamaecyparis obtus - . J. Jpn. For. Soc. 79: 222-228

Akiyo, S., Hidesgige, T., 1984. Study on the Shapes of Trees by Simulation Model. Journal of the Japanese Institute of Landscape Architecture, 47 (5)

Kikumatsu, T., Ryuzo, W., 1981. Stem Sway and Tree Form of Akamatsu, Japanese Red Pine. Journal of the Japanese Forest Society, 63 (4)

Takashi, K., Mikio, S., Takashi, Y., Michio, S., 2014 Rendering Foliage with Curved Leaves. The Institute of Image Information and Television Engineers Journal, Vol. 68, No. 11, pp. J488-J491

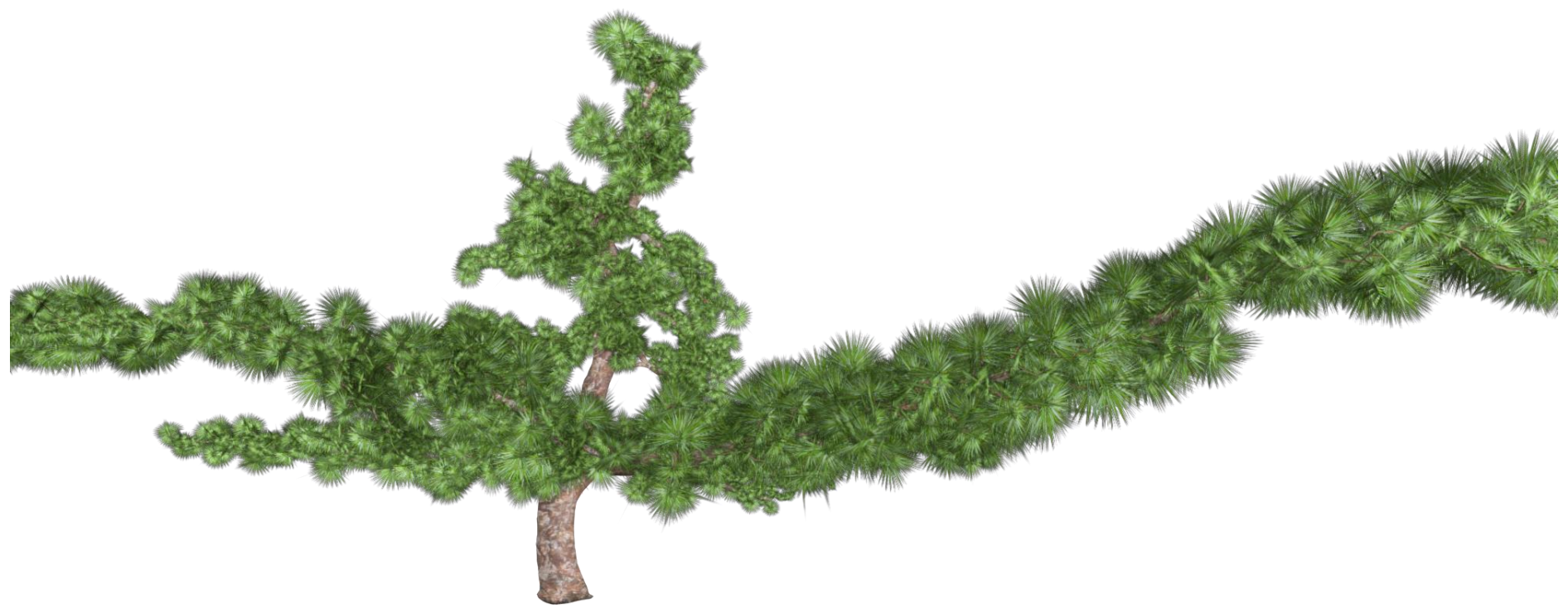

Figure 12. Successful Black Pine Model 